\title{
The automatic continuity of linear operators on some semi-prime banach algebra
}

\section{Youssef Tidli}

Sidi Mohamed Ben Abdellah University, Faculty of Sciences Dhar El Marhaz, Fes, Morroco

Email address:

youssef.tidli@gmail.com

\section{To cite this article:}

Youssef Tidli. The Automatic Continuity of Linear Operators on Some Semi-Prime Banach Algebra. Pure and Applied Mathematics Journal. Vol. 4, No. 2, 2015, pp. 43-46. doi: 10.11648/j.pamj.20150402.12

Abstract: Conditions are given for Banach algebras A and Banach algebras B which insure that every homomorphism $\mathrm{T}$ from A into B is automatic continuous. Similar results are obtained for derivations which either map the algebra A into itself.

Keywords: *-Algebra, *Prime Algebra, *-Simple Algebra, Automatic Continuity, Separting Space

\section{Introduction}

The problems of automatic continuity are the subject of a very broad theory, which uses both analytical techniques and the algebraic results. First specify what constitutes a problem of automatic continuity. Let A and B be two Banach algebras (or two topological algebras in general) and $\mathrm{T}$ is a linear map from $\mathrm{A}$ to $\mathrm{B}$; in the study of the problem of automatic continuity of $\mathrm{T}$, it is determined algebraic conditions A and/or $\mathrm{B}$, for which $\mathrm{T}$ is automatically continuous. These conditions can include application $T$. This is the case for example in the study of derivations of a Banach algebra.

In this paper, we address the problem of automatic continuity of derivation and Homomorphisms in some Banach algebras. We mainly interested in two questions:

Q1) Is any derivation in a semi-prime Banach algebra is continuous?

Q2) Is any epimorphism of a Banach algebra over a semiprime Banach algebra is continuous?

These two questions are still open even the cases of commutative Banach algebras. However, it is well known that any derivation of a semi-simple Banach algebra is continuous [8] and every epimorphism of a Banach algebra over a semi simple Banach algebra is continuous [7].

Questions (Q1) and (Q2) are asked by J. Cusack in [2]. He showed that if the answer to (Q1) or (Q2) is negative, then there exists a radical simple topologically Banach algebra non trivial.

Questions (Q1) and (Q2) are respectively equivalent to the following questions:

1) Is the ideal separting of a derivation of a Banach algebra is nilpotent?

2) Is the ideal separting an epimorphism of a Banach algebra over a Banach algebra is nilpotent?

Note that the equivalence concerning stubs ((Q1) $\Leftrightarrow 1)$ ) is given in [9]. That concerning epimorphism ((Q2) $\Leftrightarrow 1)$ ) is proved in [2].

Formulated in the commutative case (Q1) and (Q2) become:

$\left(Q^{\prime}{ }_{1}\right)$ Is all derivation in a commutative Banach algebra and semi-prime is continuous?

$\left(\mathrm{Q}_{2}^{\prime}\right)$ Is any epimorphism of a Banach algebra over a commutative Banach algebra and semi-prime is continuous?

Questions $\left(\mathrm{Q}_{1}^{\prime}\right)$ and $\left(\mathrm{Q}_{1}^{\prime}\right)$ were studied by several authors. In particular, RV Garmilla that is interested in (Q'1) (see [3], [4], [5]) and V. Runde has given in [10] some results on these two issues.

For his part, J. Cusack in [2] has shown that the following are equivalent:

i) The separting space an epimorphism of a Banach algebra over a Banach algebra is nilpotent.

ii) any epimorphism of a Banach algebra over a semiprime Banach algebra is continuous.

iii) Any epimorphism of a Banach algebra over a prime Banach algebra is continuous.

For derivations, we also have, according to [9], the equivalence of the following assertions:

i) The separting ideal in a derivation of a Banach algebra is nilpotent.

ii) Any derivation of a semi-prime Banach algebra is continuous.

iii) Any derivation of a prime Banach algebra is 
continuous.

This allows to study questions (Q1) and (Q2) for the Banach prime algebras. In the commutative case, the study is reduced to the integrity algebras.

On the other hand, RV Garmilla shown in [4] the following result in the commutative case:

Theorem 1.1 (Following [4])

Let $\mathrm{A}$ be a integral commutative Banach algebra satisfying the two conditions:

i) There is a sequence in $A\left(I_{n}\right)_{n}$ nonzero ideals such that $\cap_{n} \mathrm{I}_{\mathrm{n}}=\{0\}$.

ii) The family of closed prime ideals of $A$ is at most countable.

So all derivation in $\mathrm{A}$ is continuous.

In view of addressing the issues (Q'1) and (Q'2) simultaneously. V. Runde uses other techniques (particularly accessible ideals) and shows the following result:

Theorem 1.2 (Following [10])

Let $\mathrm{A}$ be a integral commutative Banach algebra satisfying the two conditions:

$\left.i^{\prime}\right)$ exists in a non-zero closed ideal I and an element a $\in A$ $(\mathrm{a} \neq 0)$ ) such that $\cap_{n \geq 1} \mathrm{a}^{\mathrm{n}} \mathrm{I}=\{0\}$

ii ') All prime ideals of $A$ are closed.

So all derivation in A and every epimorphism of a Banach algebra $\mathrm{B}$ on $\mathrm{A}$ is continuous

\section{Preliminaries}

Definition 2.1

$\mathrm{A}$ involution * on an algebra $\mathrm{A}$ is a map *: $\mathrm{A} \rightarrow \mathrm{A}$ satisfying the following properties.

i) $\left(\mathrm{x}^{*}\right)^{*}=\mathrm{x}$ for all $\mathrm{x}$ in $\mathrm{A}$.

ii) $(x+y)^{*}=x^{*}+y^{*}$, for all $x, y$ in $A$.

iii) $(\mathrm{x} \mathrm{y})^{*}=\mathrm{y}^{*} \mathrm{x}^{*}$, for all $\mathrm{x}, \mathrm{y}$ in $\mathrm{A}$.

iv) $1^{*}=1$,

v) $(\lambda \mathrm{x})^{*}=\bar{\lambda} \mathrm{x}^{*} \forall \lambda \in \mathrm{IK}, \mathrm{x} \in \mathrm{A}$.

This is also called an involutive algebra; We write *algebra. An elements such that $\mathrm{x}^{*}=\mathrm{x}$ are called self-adjoint.

An ideal and sub algebra, with the requirement to be *invariant: $x \in I \Rightarrow x^{*} \in I$ and so on.

As a result $\mathrm{I} * \subset \mathrm{I}$ (and then $\mathrm{I}=\mathrm{I} *$ ). It follows that all * ideal is ideals. Also if the only*-ideals of A are contained in I are $(0)$ and I then said that I was * -minimal. Note that if I is not zero * -ideal of $\mathrm{A}$, then induces an involution on $\mathrm{A} / \mathrm{I}$, also noted $*$, defined by: $(\mathrm{a}+\mathrm{I}) *=\mathrm{a} *+\mathrm{I}$. an $*$-ideal is said *- maximal if the only* -ideals containing $\mathrm{M}$ are $\mathrm{M}$ and $\mathrm{A}$. algebra is said to be simple if the only ideals of $A$ are $(0)$ and A. in case admits an involution *, we say that a is *-simple if the only * -ideals of A are (0) and A. Note that if A simple algebra equipped with an involution *, then $\mathrm{A}^{*}$-simple, but the converse is not true in general.

Example 2.1

Let $\mathrm{A}$ be an algebra and $\mathrm{A}^{\circ}$ the opposite algebra $\mathrm{A}$, then consider algebra $\mathrm{B}=\mathrm{A} \times \mathrm{A}^{\circ}$ equipped with Exchange involution $*(\mathrm{x}, \mathrm{y})=(\mathrm{y}, \mathrm{x})$. So a simple check shows that $\mathrm{B}$ is an algebra * -simple but is not simple.

Let I be a minimal left ideal of a semi-prime algebra A, then there is a minimal idempotent $\mathrm{e} \in$ A such that $\mathrm{I}=$ Ae.

Recall the radical of Jacobson, $\operatorname{Rad}(\mathrm{A})$ of an algebra $\mathrm{A}$ is defined as the intersection of all maximal left ideals of A. more If $\mathrm{A}$ is an *-algebra, then the $\mathrm{A} *$-radical , noted $\operatorname{Rad}_{*}(\mathrm{~A})$, is the intersection of all ideals *-maximal of $\mathrm{A}$. In addition, if $\operatorname{Rad}_{*}(\mathrm{~A})=(0)$, then $\mathrm{A}$ is said *-semi simple.

\section{Definition 2.2}

Let $\mathrm{T}$ a linear application of a Banach space $\mathrm{X}$ in a Banach space $\mathrm{Y}$. Then, the separating space $\sigma(\mathrm{T})$ of $\mathrm{Y}$ is the subset of Y defined by:

$\sigma(\mathrm{T})=\left\{\mathrm{y} \in \mathrm{Y} / \exists\left(\mathrm{x}_{\mathrm{n}}\right)_{\mathrm{n}} \subset \mathrm{X}: \mathrm{x}_{\mathrm{n}} \longrightarrow\|\|^{\longrightarrow} 0\right.$ et $\left.\mathrm{T}\left(\mathrm{x}_{\mathrm{n}}\right) \stackrel{\|\|}{\longrightarrow} \mathrm{y}\right\}$.

Proposition 2.1 (Following [11])

Let $\mathrm{T}$ a linear application of a Banach space $\mathrm{X}$ in a Banach space $\mathrm{Y}$. Then, $\mathrm{T}$ is continuous if, and only if, $\sigma(\mathrm{T})=(0)$.

Proposition 2.2 (Following [11])

The separting space an epimorphism of a Banach algebra A in a Banach algebra B is closed ideal.

Proposition 2.3 (Following [11])

If $\mathrm{T}$ is an epimorphism of a Banach algebra A on a Banach algebra $B$ and $b \in \sigma(T)$, then $0 \in \operatorname{Sp}(b)$.

\section{Characterizations of *-Prime Algebra}

Throughout this work, the algebras considered are supposed to be complex, associative, unital and not necessarily commutative.

A ideal I of an algebra $A$ is prime if for all elements $a, b \in$ A, we have: $a A b \subseteq I \Rightarrow a \in I$ or $b \in I$. A is semi-prime if, for all $\mathrm{a} \in \mathrm{A}$, we have: $\mathrm{aAa} \subseteq \mathrm{I} \Rightarrow \mathrm{a} \in \mathrm{I}$. The An algebra $\mathrm{A}$ is called prime (resp. Semi-prime) if the ideal $\{0\}$ is the prime (resp. Semi-prime). Let $\mathrm{M} \subseteq \mathrm{A}$. $\mathrm{M}$ is said to nil if any element of $M$ is nilpotent. $M$ is called nilpotent if there exists $\mathrm{n} \in \mathrm{IN} *$ as $\mathrm{M}^{\mathrm{n}}=\{0\}$ (the product of any $\mathrm{n}$ elements of $\mathrm{M}$ is zero). We call Prime radical (or nil radical); At the intersection of all prime ideals of $\mathrm{A}$. This radical will be noted throughout the rest $\mathrm{L}$. note that $\mathrm{L}$ is a semi-prime ideal which is contained in all semi-prime ideal of $\mathrm{A}$. Thus, $\mathrm{A}$ is semi-prime if and only if $\mathrm{L}=\{0\}$. We also show that $\mathrm{L}$ is a nil ideal containing all left (or right) nilpotent ideals. In the commutative case that $\mathrm{A}$ is prime is equivalent to saying that $\mathrm{A}$ is integral, and $\mathrm{L}$ is none other than the set of nilpotent elements of $\mathrm{A}$. We also have the following important result that achieves the prime ideals.

Proposition 3.1 (Following [6])

Let $\mathrm{I}$ be an ideal of an algebra $\mathrm{A}$ and let $\mathrm{S}$ be a subset of $\mathrm{A}$ stable multiplication as $I \cap S=\{0\}$. Then there exists a prime ideal $\mathrm{P}$ in A such that $\mathrm{P} \cap \mathrm{S}=\{0\}$.

Let $\mathrm{E}$ be a part of an algebra $\mathrm{A}$ is called the left annihilator of $E$, denoted $G(E)$, the set defined by $G(E)=\{a \in A / a E=$ $\{0\}\}$. Similarly, the right annihilator is set to of $E$ defined by $\mathrm{D}(\mathrm{E})=\{\mathrm{a} \in \mathrm{A} / \mathrm{Ea}=\{0\}\}$. We note Ann $(\mathrm{E})=\mathrm{G}(\mathrm{E}) \cap \mathrm{D}$ (E).

\section{Definition 3.1}

Let $\mathrm{A}$ be an algebra. An ideal $\mathrm{P}$ of $\mathrm{A}$ is said *-prime (resp. *-semi Prime) when for two * -idéaux I and J of A such that $\mathrm{IJ} \subset \mathrm{P}\left(\right.$ resp. $\left.\mathrm{I}^{2} \subset \mathrm{P}\right)$, then $\mathrm{I} \subset \mathrm{P}$ or $\mathrm{J} \subset \mathrm{P}($ resp. $\mathrm{I} \subset \mathrm{P})$. 
The algebra $\mathrm{A}$ is said *-Prime (resp. ${ }^{*}$-semi Premiere) if $\{0\}$ is *-prime (resp. $\{0\}$ is *-semi-prime). If $\mathrm{P}$ is a prime $*$-ideal of $\mathrm{A}$, then $\mathrm{P}$ is $*$-prime $\mathrm{A}$. Note that if $\mathrm{A}$ is a prime algebra provided with an involution *, then $\mathrm{A}$ is *-prime. However, the converse is not always true. This is the case of the following example:

Example 3.1

Let $\mathrm{A}$ be a prime algebra and $\mathrm{A}^{\circ}$ the opposite algebra $\mathrm{A}$. Consider the algebra $\mathrm{B}=\mathrm{A} \oplus \mathrm{A}$. with the exchange involution defined by $*(x, y)=(y, x), B$ is *-prime algebra which is not prime. Indeed: Let $I$ and $J$ of two ideals of $B$ as $\mathrm{IJ}=\{0\}$. Then, $(\mathrm{I} \cap \mathrm{A})(\mathrm{J} \cap \mathrm{A})=\{0\} \subset \mathrm{A}$. But A is *-Prime, hence $(I \cap A)=\{0\}$ or $(J \cap A)=\{0\}$. For example, suppose $(\mathrm{I} \cap \mathrm{A})=\{0\}$. Then $\mathrm{A}^{\circ} \mathrm{I}=(\mathrm{IA})^{*}=\{0\} \subset \mathrm{A}$. Since $\mathrm{A}$ is a prime ideal of $\mathrm{B}$, that $\mathrm{I} \subset \mathrm{A}$, we have also, $\mathrm{IA}=\{0\} \subset \mathrm{A}^{\circ}$. Since $A^{\circ}$ is a prime ideal of $B, I \subset A^{\circ}$. Therefore, $I \subset A \cap A^{\circ}$ $=\{0\}$.

It is therefore natural to ask under what conditions the converse is true. It is subject to the following proposition:

Proposition 3.2

Let A *-Prime algebra. If involution is anisotropic, then A is prime.

The involution is anisotropic if for all a in A, were:

$$
\mathrm{a} * \mathrm{a}=0 \Rightarrow \mathrm{a}=0 \text {. }
$$

\section{Proof}

Let $\mathrm{I}$ and $\mathrm{J}$ be two ideals of A such that $\mathrm{IJ}=\{0\}$. Then: (I $\left.\cap \mathrm{I}^{*}\right)\left(\mathrm{J} \cap \mathrm{J}^{*}\right)=\{0\}$. As A is *-Prime, hence $(\mathrm{I} \cap \mathrm{I} *)=\{0\}$ or $\left(\mathrm{J} \cap \mathrm{J}^{*}\right)=\{0\}$. Suppose $\left(\mathrm{I} \cap \mathrm{I}^{*}\right)=\{0\}$. So for all a in $\mathrm{I}$, we have: aa* $=0$. As $*$ is anisotropic, $\mathrm{a}=0 \forall \mathrm{a} \in \mathrm{A}$. As a result $\mathrm{I}=\{0\}$.

In the following, we give some characterizations *-Prime algebras.

\section{Proposition 3.3}

Let $\mathrm{A}$ be an algebra and $\mathrm{P}$ prime sub-algebra (resp. Semiprime sub-algebra) of $A$. Then, $G(P)=D(P)=A n n(P)$ is minimal prime ideal (resp. semi-prime ideal) of A and Ann (P) is the only complementary to right and left of P. Moreover, if Ann (A) $\neq\{0\}$, then there is no ideal prime strictly content in $\mathrm{P}$.

\section{Proof}

Let $\mathrm{I}$ be a right ideal of A such that PI $=\{0\}$. Then, IP is a right ideal of $\mathrm{P}$ and (IP) ${ }^{2}=\{0\}$. Hence, $\mathrm{IP}=\{0\}$, through $\mathrm{D}(\mathrm{P})$ $\subseteq \mathrm{G}(\mathrm{P})$. Similarly, it is shown that $\mathrm{G}(\mathrm{P}) \subseteq \mathrm{D}(\mathrm{P})$. Therefore, $\mathrm{D}(\mathrm{P})=\mathrm{G}(\mathrm{P})=$ Ann $(\mathrm{P})$. If $\mathrm{I}$ is complementary right ideal $\mathrm{P}$, then $\mathrm{IP} \subseteq \mathrm{I} \cap \mathrm{P}=\{0\}$, where $\mathrm{I} \subseteq$ Ann $(\mathrm{P})$, which implies that Ann (P) is the only complementary to the right of P. Similarly, it is shown Ann (P) is the only complementary left of P. Let I and $\mathrm{J}$ be two ideals of A such that $\mathrm{IJ} \subseteq$ Ann $(\mathrm{P})$. then PIJ $=\{0\}$, hence JPI $\subseteq \mathrm{P}$ and $(\mathrm{JPI})^{2}=\{0\}$. As $\mathrm{P}$ is a prime sub-algebra, JPI $=\{0\}$, therefore $\{0\}=(\mathrm{PJ})(\mathrm{PI}) \subseteq \mathrm{P}$. whereas the result, $\mathrm{PJ}$ $=\{0\}$ or $\mathrm{PI}=\{0\}$, where Ann $(\mathrm{P})$ is a prime ideal of $\mathrm{A}$. Suppose now that $\mathrm{Q}$ is prime ideal of $\mathrm{A}$ such that $\mathrm{Q} \subset \mathrm{Ann}(\mathrm{P})$. So $\mathrm{P}$ Ann $(\mathrm{P})=\{0\} \subset \mathrm{Q}$, which is absurd. So Ann $(\mathrm{P})$ is a minimal prime ideal. By similar reasoning, we easily verify that if $A n n(P) \neq\{0\}$, then there is no prime ideal of A strictly contained in P. For the semi-prime case, the reasoning is similar to the prime case.
Lemma 3.1 (Lemma Andrunakiewich) Let A be a ring, I an ideal of $\mathrm{A}$ and $\mathrm{J}$ an ideal of $\mathrm{I}$. Then $\langle\mathrm{J}\rangle^{3}=\mathrm{I}$, where $<\mathrm{J}>$ is the ideal of A generated by $\mathrm{J}$.

Proposition 3.4

Let $\mathrm{A}$ be an algebra and I, $\mathrm{P}$ two ideals of $\mathrm{A}$ such that $\mathrm{P}$ is prime, $I \neq\{0\}$ et $P \cap I=\{0\}$. So we have: 1) I is a prime subalgebra which contains no prime ideal of A. 2) $\mathrm{P}$ is a minimal prime ideal of $\mathrm{A}$ and $\mathrm{P}=\mathrm{Ann}(\mathrm{I})$.

Proof

1) Let $\mathrm{H}, \mathrm{K}$ two ideals $\mathrm{I}$ as $\mathrm{HK}=\{0\}$. By Lemma of Andrunakiewich, $<\mathrm{H}>^{3}<\mathrm{K}>^{3} \subseteq \mathrm{HK}=\{0\} \subseteq \mathrm{P}$, where $<\mathrm{H}>$ (resp. $<\mathrm{K}>$ ) is the ideal of $\mathrm{A}$ generated by $\mathrm{H}$ (resp. $\mathrm{K}$ ). We have $\mathrm{P}$ is a prime ideal, hence $<\mathrm{H}>\subseteq \mathrm{P} \cap \mathrm{I}=\{0\}$ or $<\mathrm{K}>\subseteq$ $\mathrm{P} \cap \mathrm{I}=\{0\}$. Therefore, $\mathrm{I}$ is a prime sub-algebra. If $\mathrm{Q}$ is an prime ideal strictly content in $\mathrm{I}$, then $\mathrm{PI}=\{0\} \subseteq \mathrm{Q}$, which is absurd. 2) Follows immediately from 1) and the previous proposition.

Proposition 3.5

Let A be a *-prime *-algebra no prime. Then there exists a prime ideal $P$ such that:

1) $\mathrm{P} \cap \mathrm{P} *=\{0\}$.

2) $\mathrm{P}$ and $\mathrm{P} *$ are two minimal prime ideals, $\mathrm{P}=\operatorname{Ann}(\mathrm{P} *)$ and Ann $\mathrm{P} *=\operatorname{Ann}(\mathrm{P})$.

Proof

1) We apply Zorn's lemma to the set D of ideals of A such that $\mathrm{I} \cap \mathrm{I}^{*}=\{0\}$. Then $\mathrm{D}$ has a maximal element $\mathrm{P}$ such that $\mathrm{P} \cap \mathrm{P} *=\{0\}$. Let I, J be two ideals of A such that IJ $\subseteq \mathrm{P}$. Then, $(\mathrm{I} \cap \mathrm{I} *)\left(\mathrm{J} \cap \mathrm{J}^{*}\right)(\mathrm{I} \cap \mathrm{I} *)=\{0\}$. This implies that $\mathrm{I} \cap \mathrm{I}^{*}=\{0\}$ or $\mathrm{J} \cap \mathrm{J}^{*}=\{0\}$. Since $\mathrm{P}$ is maximum in $\mathrm{D}$, then $\mathrm{I} \subseteq \mathrm{P}$ or $\mathrm{J} \subseteq \mathrm{P}$.

2)Follows immediately from the previous proposition.

Proposition 3.6

Let $\mathrm{A}$ be an *-algebra. Consider the following assertion:

1) A is *-simple

2) $\mathrm{A}$ is *-Prime

3) $\mathrm{A}$ is -*semi prime

4) A is semi-prime. Then: 1) $\Rightarrow$ 2) $\Rightarrow$ 3) $\Rightarrow 4$ ).

Proof

1) $\Rightarrow 2$ ) Let I and J two non zero *-idéaux of A. Since A is *-simple then $\mathrm{IJ}=\mathrm{A}^{2} \neq\{0\}$.

2) $\Rightarrow$ 3) obvious.

$3) \Rightarrow 4$ ) Let I be a left ideal of A such that $I^{2}=\{0\}$. Then, $\left(\mathrm{I} \cap \mathrm{I}^{*}\right)^{2}=\{0\}$. Since $\mathrm{A}$ is* -Prime, $\mathrm{I} \cap \mathrm{I} *=\{0\}$. On the other hand $(\mathrm{I}+\mathrm{I} *)^{2}=\mathrm{I}^{2}+\mathrm{II} * \mathrm{I} * \mathrm{I}+(\mathrm{I} *)^{2}=(\mathrm{I} *)^{2}=\left(\mathrm{I}^{2}\right)^{*}$ $=\{0\}$. As a result, $\mathrm{I} \subseteq \mathrm{I}+\mathrm{I} *=\{0\}$.

Remark 3.1

From the above proposition, we see that the concept of *semi prime and semi prime on A coincides, which gives an equivalent definition of semi-prime * -algebras, using a particular class ideals instead to considered all left ideals of A.

\section{Automatic Continuity}

In this work, we study the questions (Q1) and (Q2) in the class of *-Prime Banach *-algebra. We show that if a B is a *-prime Banach algebra that is not prime, then all surjective Homomorphism (or dense range) of an Banach algebra A over B is continuous (Theorem 4.1). Similarly, any derivation 
in B is continuous (Theorem 4.2). In particular, if A is a *prime Banach algebra not prime, then all A complete normed on $\mathrm{A}$ are equivalent.

Theorem 4.1 Let $\mathrm{T}$ be a homomorphism of Banach algebra A on a Banach *-algebra B. If B is *-prime not Prime and if $\mathrm{T}$ is surjective (or dense range), then $\mathrm{T}$ is continuous.

Proof

Since $\mathrm{B}$ is a *-prime not Prime algebra, there is a minimal prime nonzero $\mathrm{P}$ such that $\mathrm{P} \cap \mathrm{P} *=\{0\}$ and $\mathrm{P}=\operatorname{Ann}(\mathrm{P} *), \mathrm{P}$ $*=$ Ann (P) Proposition (5). Let $\sigma(T)$ be the ideal separting in B. Suppose $\sigma(\mathrm{T}) \not \subset \mathrm{P}$, then $\mathrm{P}$ is a closed ideal [2]. On the other hand, if $\mathrm{p}$ is a nonzero element of $\mathrm{P}$, then $\overline{p \sigma(T)}=$ $\sigma(T)$ (stability lemma [11]). Therefore: $p \sigma(T) \subseteq P$, then $\overline{p \sigma(T)}=\sigma(T) \subseteq \bar{P}=P$. Which contradicts the assumption. Following $\sigma(T) \subseteq \mathrm{P}$. By the same reasoning, we show that $\sigma(T) \subseteq \mathrm{P}^{*}$. Which gives, $\sigma(T) \subseteq \mathrm{P} \cap \mathrm{P}^{*}=\{0\}$. Therefore, $\mathrm{T}$ is continuous (following [proposition 2.1]).

Theorem 4.2 All derivation in $\mathrm{a}^{*}$-prime not prime Banach algebra is continuous.

Proof

Same proof as the previous theorem, since the separting space of a derivation in Banach algebra $\mathrm{A}$ is an ideal of $\mathrm{A}$.

Corollary 4.1 Let (A, \|. \|) be a* -Prime Banach algebra. Then, we have:

i) All complete norm on A are equivalent.

ii) The involution * is continuous.

Proof

i) It is enough to apply the previous theorem to the Identity of A.

ii) That is to say the linear Application q of A in IR defined by $\mathrm{q}(\mathrm{x})=\|\mathrm{x} *\|(\mathrm{x} \in \mathrm{A})$.

We easily verify that $\mathrm{q}$ is a complete norm on A. And according to $\mathrm{i}$ ), $\mathrm{q}$ is equivalent to $\|$. $\|$

\section{References}

[1] F.F. Bonsall and J. Duncan, Complete normed algebras, Spring6 Verlag. Berlin (1973).

[2] J. Cusack, Automatic Continuity and topologically simple Banach algebras, J. London. Math. Soc (2), 16 (1977), 439-500.

[3] R. V. Garimella, Continuity of derivation on some semi-prime Banach algebra, Proc. Amer. Math. Soc., 99 (1987), 289 [12]292.

[4] R.V. Garimella, Prime ideals and the continuity of derivation on integral domins, Indian. J. Pure app. Math, 22 (12) (1991), 1013-1018.

[5] R.V. Garimella, On nilpotenty of the separting ideal of a derivation. Proc. Amer. Math. Soc., 117, (1993), 167-174.

[6] N. Jacobson, Basic Algebra II, W. H. Ferman and Compagny (1980).

[7] B.E Johnson, The uniqueness of the (complete) norm topology, Bull. Amer. Math. Soc. 73, (1967) 537-539

[8] B.E Johnson, and A. M. Sinclair, Continuity of derivation and a problem of Kaplansky, Amer. J. Math. Math. 90 (1968), 1067-1073.

[9] M. Mathieu et V. Runde, Derivations mapping into the radical II, Bull. London Math. Soc., 24(1992), 485-487.

[10] V. Runde, Continuity of derivations and epimorphisms, Pacific J. of Math., Vol. 147, Nr. 2 (1991), 365-374.

[11] A. M. Sinclair, Automatic Continuity of linear operators, London. Math. Soc. Lecture Note Series 21 (C.U.P., Combridge 1976).

[12] Y. TIDLI, L. OUKHTITE, A. TAJMOUATI, On the Automatic continuity of the epimorphisms in *algebras of Banach. IJMMS 2004: 22 1183-1187 (2002). 